\title{
ON THE RESIDUAL AND INACTIVITY TIMES OF THE COMPONENTS OF USED COHERENT SYSTEMS
}

\author{
S. GOLIFORUSHANI *** AND \\ M. ASADI,**** University of Isfahan \\ N. BALAKRISHNAN, ${ }^{* * * *}$ McMaster University
}

\begin{abstract}
In the study of the reliability of technical systems in reliability engineering, coherent systems play a key role. In this paper we consider a coherent system consisting of $n$ components with independent and identically distributed components and propose two time-dependent criteria. The first criterion is a measure of the residual lifetime of live components of a coherent system having some of the components alive when the system fails at time $t$. The second criterion is a time-dependent measure which enables us to investigate the inactivity times of the failed components of a coherent system still functioning though some of its components have failed. Several ageing and stochastic properties of the proposed measures are then established.
\end{abstract}

Keywords: $k$-out-of- $n$ system; hazard rate; reversed hazard rate; stochastic ordering; residual lifetime; inactivity time; order statistics; reliability; signature; conditional lifetime; increasing failure rate

2010 Mathematics Subject Classification: Primary 60E15

Secondary 60K 10

\section{Introduction}

Reliability properties of coherent systems have been discussed quite extensively in the literature. By definition, a technical structure consisting of $n$ components is said to be a coherent system if there is no irrelevant component in the system (a component is said to be irrelevant if its performance does not affect the performance of the system) and it is monotone with respect to each component (that is, when a failed component in the system is replaced by a working one, the system cannot get any worse). Some important special cases of coherent systems of order $n$ are $(n-k+1)$-out-of- $n$ systems, $k=1,2, \ldots, n$, in which the cases $k=1$ and $k=n$ correspond to the series and parallel systems, respectively.

Several authors have studied the residual lifetime and inactivity time of the coherent systems in different scenarios. See, for example, Bairamov et al. [6], Asadi and Bayramoglu [2], Asadi and Bayramoglu [3], Khaledi and Shaked [8], Asadi and Goliforushani [5], Li and Zhang [11], Navarro and Hernandez [15], Samaniego et al. [22], and Zhang [26]. The inactivity times of a component or a system have also been discussed by Navarro et al. [17], Asadi and Berred [4],

Received 25 November 2010; revision received 24 October 2011.

* Postal address: Department of Statistics, University of Isfahan, Isfahan, 81744, Iran.

** Email address: sareh.goli@stat.ui.ac.ir

*** Email address: m.asadi@sci.ui.ac.ir

**** Postal address: Department of Mathematics and Statistics, McMaster University, 1280 Main Street West Hamilton, Ontario L8S 4K1, Canada. Email address: bala@univmail.cis.mcmaster.ca 
Asadi [1], Tavangar and Asadi [24], Khaledi and Shaked [8], Li and Zhao [13], Li and Zhang [11], [12], Navarro et al. [20], and Gertsbakh et al. [7].

In the study of the reliability of coherent systems, a useful tool is the concept of 'signature'. The signature vector of a system enables one to represent the distribution function of the lifetime of a coherent system in terms of the distribution functions of ordered lifetimes of its components. Consider a coherent system consisting of $n$ components with independent and identically distributed (i.i.d.) lifetimes $X_{1}, X_{2}, \ldots, X_{n}$ which are distributed according to a common continuous distribution $F$. Let $T=T\left(X_{1}, X_{2}, \ldots, X_{n}\right)$ be the system's lifetime. The signature of the system is defined to be a probability vector $s=\left(s_{1}, s_{2}, \ldots, s_{n}\right)$ in which

$$
s_{i}=\mathrm{P}\left(T=X_{i: n}\right), \quad i=1,2, \ldots, n,
$$

where $X_{i: n}$ denotes the $i$ th ordered lifetime of the components. It is well known that the signature vector $s$ does not depend on the underlying distribution function $F$. This fact leads to the following theorem, which shows that the distribution function of the system lifetime can be represented as a mixture of the distribution functions of the ordered lifetimes of its components where the mixing distribution is the probability vector $\boldsymbol{s}$ (see [9] and [21]).

Theorem 1.1. If $\bar{F}_{T}(t)$ denotes the survival function of the system lifetime then

$$
\bar{F}_{T}(t)=\sum_{i=1}^{n} s_{i} \bar{F}_{X_{i: n}}(t),
$$

where $\bar{F}_{X_{i: n}}(t)$ denotes the survival function of $X_{k: n}$.

Recently, the reliability properties of a coherent system based on the properties of its signature have been extensively discussed. For some recent developments on this subject, we refer the reader to [9], [16], [17], [18], [19], [22], and [25].

In this paper we consider coherent systems for which the signature vector is of one of the following forms.

(A) $s=\left(s_{1}, \ldots, s_{i}, 0, \ldots, 0\right)$, where $s_{k}>0$ for $k=1,2, \ldots, i, i=1,2, \ldots, n-1$.

(B) $s=\left(0, \ldots, 0, s_{i+1}, \ldots, s_{n}\right)$, where $s_{k}>0$ for $k=i+1, i+2, \ldots, n, i=1,2, \ldots, n$.

A coherent system with signature of the form (A) has the property that, upon failure of the system at time $t$, components of the system with lifetimes $X_{k: n}, k=i+1, i+2, \ldots, n$ are still alive. Hence, after the failure of the system, they can be removed from the system and used for other testing purposes. Thus, the study of the reliability properties of such components may be of interest for engineers and system designers. On the other hand, a coherent system with signature vector of the form (B) has the property that, when the system fails at time $t$, it has to be due to the failure of one of the components with lifetime $X_{k: n}, k=i+1, i+2, \ldots, n$.

In the sequel, we define two conditional probabilities. Assuming that the signature vector of the system is of the form (A), we define

$$
P_{j, T}(t, x)=\mathrm{P}\left(X_{j: n}-t>x \mid T<t<X_{j: n}\right), \quad x, t>0, j=i+1, i+2, \ldots, n .
$$

In an analogous manner, for the system having a signature vector of the form (B), we define

$$
P_{j, T}^{*}(t, x)=\mathrm{P}\left(t-X_{j: n}>x \mid X_{j: n}<t<T\right), \quad x, t>0, j=1,2, \ldots, i .
$$


The conditional probability in (1.1) gives the reliability of the residual lifetime of a live component with lifetime $X_{j: n}, k=i+1, i+2, \ldots, n$, when the system has failed before time $t$ while the component with lifetime $X_{j: n}$ is still alive at time $t$. The conditional probability in (1.2) gives the survival function of the inactivity time of a component with lifetime $X_{j: n}, j=$ $1, \ldots, i$, under the condition that the system is functioning at time $t$ but the component with lifetime $X_{j: n}$ has already failed in the system. A system with signature vector of the form (B) can be considered as a 'black box' in the sense that, when the system is functioning, upon the failure of $X_{j: n}, j=1,2, \ldots, i$, the failure time of that component is unknown. Hence, the study of the conditional probability in (1.2) is important for system designers to get some useful information on the inactivity times of the failed components.

The rest of this paper is organized as follows. In Section 2 we concentrate on the conditional probability in (1.1). We begin by obtaining the functional form of $P_{j, T}(t, x)$ and show that it can be represented as a mixture of the residual lifetimes of the live components of the system with simpler structures. Among the results in this section, we prove that when the common hazard rate of the components of the system is increasing, then $P_{j, T}(t, x)$ is a decreasing function of time. We also prove that when two coherent systems with the same structure have components with distribution functions $F$ and $G$, respectively, for which the hazard rates of the components are ordered, then the corresponding residual lives of the systems are also ordered. In Section 3 we focus on the reliability function $P_{j, T}^{*}(t, x)$ defined in (1.2). We prove that if in a coherent system with signature vector of the form (B) the reversed hazard rate of the components of the system is decreasing in time, then $P_{j, T}^{*}(t, x)$ is an increasing function of time. Stochastic comparisons between the inactivity times of the failed components of two different systems are also made under the condition that the components of the systems are ordered in terms of their reversed hazard rates.

Before proceeding to present the main results, we recall some stochastic order definitions for two nonnegative random variables $X$ and $Y$ having distribution functions $F$ and $G$ and probability density functions $f$ and $g$, respectively.

- $X$ is said to be less than $Y$ in the stochastic order, denoted by $X \leq_{\text {st }} Y$, if $\bar{F}(x) \leq$ $\bar{G}(x), x>0$, where $\bar{F}=1-F$ and $\bar{G}=1-G$.

- $X$ is said to be less than $Y$ in the hazard order, denoted by $X \leq \mathrm{hr} Y$, if $\bar{F}(x) / \bar{G}(x)$ is a decreasing function of $x$. If the densities of $X$ and $Y$ exist, $X \leq_{\mathrm{hr}} Y$ is equivalent to saying that $h_{1}(t) \geq h_{2}(t)$, where $h_{1}(t)=f(t) / \bar{F}(t)$ and $h_{2}(t)=g(t) / \bar{G}(t)$ denote the hazard rates of $X$ and $Y$, respectively.

- $X$ is said to be less than $Y$ in the reversed hazard order, denoted by $X \leq_{\text {rh }} Y$, if $F(x) / G(x)$ is a decreasing function of $x$. If the densities of $X$ and $Y$ exist, $X \leq_{\mathrm{rh}} Y$ is equivalent to saying that $r_{1}(t) \leq r_{2}(t)$, where $r_{1}(t)=f(t) / F(t)$ and $r_{2}(t)=g(t) / G(t)$ denote the reversed hazard rates of $X$ and $Y$, respectively.

- $X$ is said to be less than $Y$ in the likelihood ratio order, denoted by $X \leq_{\operatorname{lr}} Y$, if $f(x) / g(x)$ is a decreasing function of $x$.

\section{Residual lifetimes of the live components}

Let $T$ be the lifetime of a coherent system of order $n$, and let $X_{1}, X_{2}, \ldots, X_{n}$ be the lifetimes of its components. We assume that $X_{1}, X_{2}, \ldots, X_{n}$ are i.i.d. according to a common underlying continuous distribution $F$. Furthermore, we assume that the signature of the system has the 
TABLE 1: Coherent systems with four components and signatures of the form (2.1).

\begin{tabular}{cc}
\hline$T=T\left(X_{1}, X_{2}, X_{3}, X_{4}\right)$ & Signature \\
\hline$X_{2: 2}=\min \left(X_{1}, X_{2}\right)(2$-series $)$ & $\left(\frac{1}{2}, \frac{1}{3}, \frac{1}{6}, 0\right)$ \\
$\min \left(X_{2}, \max \left(X_{1}, X_{3}\right)\right)($ consecutive 2-out-of-3: $F)$ & $\left(\frac{1}{4}, \frac{5}{12}, \frac{1}{3}, 0\right)$ \\
$\min \left(X_{1}, \max \left(X_{2}, X_{3}\right), \max \left(X_{3}, X_{4}\right)\right)$ & $\left(\frac{1}{4}, \frac{7}{12}, \frac{1}{6}, 0\right)$ \\
$\min \left(X_{1}, \max \left(X_{2}, X_{3}, X_{4}\right)\right)(3$-parallel) & $\left(\frac{1}{4}, \frac{1}{4}, \frac{1}{2}, 0\right)$ \\
$\min \left(X_{1}, X_{2}, X_{3}\right)$ & $\left(\frac{3}{4}, \frac{1}{4}, 0,0\right)$ \\
$\max \left(\min \left(X_{1}, X_{2}, X_{3}\right), \min \left(X_{2}, X_{3}, X_{4}\right)\right)($ consecutive 3-out-of-4: $F)$ & $\left(\frac{1}{2}, \frac{1}{2}, 0,0\right)$ \\
$\min \left(X_{2: 3}, X_{4}\right)$ & $\left(\frac{1}{4}, \frac{3}{4}, 0,0\right)$ \\
$X_{1: 4}=\min \left(X_{1}, X_{2}, X_{3}, X_{4}\right)$ (series) & $(1,0,0,0)$ \\
\hline
\end{tabular}

form

$$
\boldsymbol{s}=\left(s_{1}, \ldots, s_{i}, 0, \ldots, 0\right), \quad 1 \leq i<n .
$$

Some examples of systems with signatures of the form given in (2.1) are presented in Table 1. Based on the structure of the system, it is clear that the components with lifetimes $X_{k: n}, k=$ $i+1, i+2, \ldots, n$, would never cause the failure of the system. That is,

$$
\mathrm{P}\left(T=X_{k: n}\right)=0, \quad k=i+1, i+2, \ldots, n .
$$

In what follows, we assume that the system has failed by time $t$. We are then interested in the following conditional probability:

$$
P_{j, T}(t, x)=\mathrm{P}\left(X_{j: n}-t>x \mid T<t<X_{j: n}\right), \quad x, t>0, j=i+1, i+2, \ldots, n .
$$

Evidently, this conditional probability represents the residual lifetimes of the components that could never fail in the system, under the condition that the system has failed by time $t$. In what follows, we first derive the form of $P_{j, T}(t, x)$. For $j \geq i+1$ and all $x, t \geq 0$, we have

$$
\begin{aligned}
\mathrm{P}\left(T<t, X_{j: n}>t+x\right) & =\sum_{k=1}^{i} \mathrm{P}\left(T=X_{k: n}, T<t, X_{j: n}>x+t\right) \\
& =\sum_{k=1}^{i} \mathrm{P}\left(T=X_{k: n}, X_{k: n}<t, X_{j: n}>x+t\right) \\
& =\sum_{k=1}^{i} \mathrm{P}\left(T=X_{k: n}\right) \mathrm{P}\left(X_{k: n}<t, X_{j: n}>x+t \mid T=X_{k: n}\right) \\
& =\sum_{k=1}^{i} s_{k} \mathrm{P}\left(X_{k: n}<t, X_{j: n}>x+t\right),
\end{aligned}
$$

where $s_{k}=\mathrm{P}\left(T=X_{k: n}\right), k=1, \ldots, i$, and the last equality is due to the fact that the events $\left\{T=X_{k: n}\right\}$ and $\left\{X_{k: n}<t, X_{j: n}>x+t\right\}$ are independent. Therefore, for all $x, t>0$, we have

$$
P_{j, T}(t, x)=\frac{\mathrm{P}\left(T<t, X_{j: n}>t+x\right)}{\mathrm{P}\left(T<t, X_{j: n}>t\right)}=\sum_{k=1}^{i} p_{k}(t) \gamma_{j, k, n}(t, x),
$$


where $\gamma_{j, k, n}(t, x)=\mathrm{P}\left(X_{j: n}-t>x \mid X_{k: n}<t<X_{j: n}\right)$,

$$
p_{k}(t)=s_{k} \frac{\mathrm{P}\left(X_{k: n}<t<X_{j: n}\right)}{\mathrm{P}\left(T<t<X_{j: n}\right)}=\mathrm{P}\left(T=X_{k: n} \mid T<t<X_{j: n}\right),
$$

and the last equality follows from [14]. The vector

$$
\boldsymbol{p}(t)=\left(p_{1}(t), \ldots, p_{i}(t), 0,0, \ldots, 0\right)
$$

can be regarded as the conditional signature of the system in which the element $p_{k}(t)$ is the probability that the component with lifetime $X_{k: n}$ causes the failure of the system given that the system has failed but the components with lifetimes $X_{k: n}, k=i+1, i+2, \ldots, n$, are still alive.

Before presenting the main results, we examine some properties of $p_{k}(t)$. The following lemma shows that $p_{k}(t)$ can be represented in terms of the ratio $\phi(t)=F(t) / \bar{F}(t)$.

Lemma 2.1. For $k=1, \ldots, i$ and $i<j$, we have

where

$$
p_{k}(t)=\frac{s_{k} W_{j, k}(t)}{\sum_{m=1}^{i} s_{m} W_{j, m}(t)},
$$

$$
W_{j, m}(t)=\sum_{l=m}^{j-1}\left(\begin{array}{l}
n \\
l
\end{array}\right)(\phi(t))^{l}
$$

Proof. We have

$$
\begin{aligned}
p_{k}(t) & =s_{k} \frac{\mathrm{P}\left(X_{k: n}<t<X_{j: n}\right)}{\mathrm{P}\left(T<t<X_{j: n}\right)} \\
& =s_{k} \frac{\mathrm{P}\left(X_{k: n}<t\right)-\mathrm{P}\left(X_{j: n}<t\right)}{\sum_{m=1}^{i} s_{m}\left\{\mathrm{P}\left(X_{m: n}<t\right)-\mathrm{P}\left(X_{j: n}<t\right)\right\}} \\
& =\frac{s_{k} \sum_{l=k}^{j-1}\left(\begin{array}{c}
n \\
l
\end{array}\right)(F(t))^{l}(1-F(t))^{n-l}}{\sum_{m=1}^{i} s_{m} \sum_{l=m}^{j-1}\left(\begin{array}{c}
n \\
l
\end{array}\right)(F(t))^{l}(1-F(t))^{n-l}} \\
& =\frac{s_{k} \sum_{l=k}^{j-1}\left(\begin{array}{c}
n \\
l
\end{array}\right)(\phi(t))^{l}}{\sum_{m=1}^{i} s_{m} \sum_{l=m}^{j-1}\left(\begin{array}{l}
n \\
l
\end{array}\right)(\phi(t))^{l}},
\end{aligned}
$$

as required.

Theorem 2.1. We have

(a) $\lim _{t \rightarrow 0} \boldsymbol{p}(t)=(1,0, \ldots, 0,0)$;

(b) $\lim _{t \rightarrow \infty} \boldsymbol{p}(t)=\boldsymbol{s}$.

Proof. To obtain $\lim _{t \rightarrow \infty} p_{k}(t)$ and $\lim _{t \rightarrow 0} p_{k}(t)$, since $\phi(t)$ is an increasing function of $t$ and $\lim _{t \rightarrow \infty} \phi(t)=\infty$ and $\lim _{t \rightarrow 0} \phi(t)=0$, without loss of generality, it is sufficient to find $\lim _{t \rightarrow \infty} g_{k}(t)$ and $\lim _{t \rightarrow 0} g_{k}(t)$, respectively, where

$$
g_{k}(t)=\frac{s_{k} \sum_{l=k}^{j-1}\left(\begin{array}{l}
n \\
l
\end{array}\right) t^{l}}{\sum_{m=1}^{i} s_{m} \sum_{l=m}^{j-1}\left(\begin{array}{l}
n \\
l
\end{array}\right) t^{l}} .
$$


However,

$$
\begin{aligned}
g_{k}(t) & =\frac{s_{k} \sum_{l=k}^{j-1}\left(\begin{array}{l}
n \\
l
\end{array}\right) t^{l}}{\sum_{l=1}^{j-1}\left(\sum_{m=1}^{\min (l, i)} s_{m}\right)\left(\begin{array}{c}
n \\
l
\end{array}\right) t^{l}} \\
& =\frac{s_{k}\left(\begin{array}{l}
n \\
k
\end{array}\right) t^{k}+s_{k}\left(\begin{array}{c}
n \\
k+1
\end{array}\right) t^{k+1}+\cdots+s_{k}\left(\begin{array}{c}
n \\
j-1
\end{array}\right) t^{j-1}}{\left(\sum_{m=1}^{\min (1, i)} s_{m}\right)\left(\begin{array}{c}
n \\
1
\end{array}\right) t+\left(\sum_{m=1}^{\min (2, i)} s_{m}\right)\left(\begin{array}{c}
n \\
2
\end{array}\right) t^{2}+\cdots+\left(\sum_{m=1}^{\min (j-1, i)} s_{m}\right)\left(\begin{array}{c}
n \\
j-1
\end{array}\right) t^{j-1}} \\
& =\frac{s_{k}\left(\begin{array}{l}
n \\
k
\end{array}\right) t^{k}+s_{k}\left(\begin{array}{c}
n \\
k+1
\end{array}\right) t^{k+1}+\cdots+s_{k}\left(\begin{array}{c}
n \\
j-1
\end{array}\right) t^{j-1}}{s_{1}\left(\begin{array}{c}
n \\
1
\end{array}\right) t+\left(\sum_{m=1}^{\min (2, i)} s_{m}\right)\left(\begin{array}{c}
n \\
2
\end{array}\right) t^{2}+\cdots+\left(\sum_{m=1}^{i} s_{m}\right)\left(\begin{array}{c}
n \\
j-1
\end{array}\right) t^{j-1}} .
\end{aligned}
$$

Now, we have

$$
\begin{aligned}
\lim _{t \rightarrow 0} g_{k}(t) & =\lim _{t \rightarrow 0} \frac{s_{k}\left(\begin{array}{l}
n \\
k
\end{array}\right) t^{k}+s_{k}\left(\begin{array}{c}
n \\
k+1
\end{array}\right) t^{k+1}+\cdots+s_{k}\left(\begin{array}{c}
n \\
j-1
\end{array}\right) t^{j-1}}{s_{1}\left(\begin{array}{c}
n \\
1
\end{array}\right) t+\left(\sum_{m=1}^{\min (2, i)} s_{m}\right)\left(\begin{array}{c}
n \\
2
\end{array}\right) t^{2}+\cdots+\left(\sum_{m=1}^{i} s_{m}\right)\left(\begin{array}{c}
n \\
j-1
\end{array}\right) t^{j-1}} \\
& = \begin{cases}\frac{s_{1}\left(\begin{array}{l}
n \\
1
\end{array}\right)}{s_{1}\left(\begin{array}{c}
n \\
1
\end{array}\right)}=1, & k=1, \\
0, & k>1,\end{cases}
\end{aligned}
$$

which completes the proof of part (a). Next, we have

$$
\begin{aligned}
\lim _{t \rightarrow \infty} g_{k}(t) & =\lim _{t \rightarrow \infty} \frac{s_{k}\left(\begin{array}{c}
n \\
k
\end{array}\right) t^{k}+s_{k}\left(\begin{array}{c}
n \\
k+1
\end{array}\right) t^{k+1}+\cdots+s_{k}\left(\begin{array}{c}
n \\
j-1
\end{array}\right) t^{j-1}}{s_{1}\left(\begin{array}{c}
n \\
1
\end{array}\right) t+\left(\sum_{m=1}^{\min (2, i)} s_{m}\right)\left(\begin{array}{c}
n \\
2
\end{array}\right) t^{2}+\cdots+\left(\sum_{m=1}^{i} s_{m}\right)\left(\begin{array}{c}
n \\
j-1
\end{array}\right) t^{j-1}} \\
& =\frac{s_{k}\left(\begin{array}{c}
n \\
j-1
\end{array}\right)}{\left(\sum_{m=1}^{i} s_{m}\right)\left(\begin{array}{c}
n \\
j-1
\end{array}\right)} \\
& =s_{k},
\end{aligned}
$$

which completes the proof of part (b).

Theorem 2.2. Let $\boldsymbol{p}(t)$ be a vector of coefficients with $p_{k}(t)$ as in (2.2). Then, $\boldsymbol{p}\left(t_{1}\right) \leq_{\mathrm{st}} \boldsymbol{p}\left(t_{2}\right)$ for all $0 \leq t_{1} \leq t_{2}$ and $\boldsymbol{p}(t) \leq_{\text {st }} \boldsymbol{s}$ for all $t \geq 0$.

Proof. To prove the required result, we need to show that, for all $i \geq u \geq 1$, we have $\sum_{k=u}^{i} p_{k}\left(t_{1}\right) \leq \sum_{k=u}^{i} p_{k}\left(t_{2}\right)$, or, equivalently, that $\sum_{m=1}^{i} \sum_{k=u}^{i} s_{k} s_{m}\left\{\bar{W}_{j, m}\left(t_{2}\right) W_{j, k}\left(t_{1}\right)-\right.$ $\left.W_{j, m}\left(t_{1}\right) W_{j, k}\left(t_{2}\right)\right\} \leq 0$, where $W_{j, n}(t)$ is as defined in (2.3). We have

$$
\begin{aligned}
\sum_{m=1}^{i} \sum_{k=u}^{i} s_{k} s_{m}\left\{W_{j, m}\left(t_{2}\right) W_{j, k}\left(t_{1}\right)-W_{j, m}\left(t_{1}\right) W_{j, k}\left(t_{2}\right)\right\} \\
=\sum_{m=1}^{u-1} \sum_{k=u}^{i} s_{k} s_{m}\left\{W_{j, m}\left(t_{2}\right) W_{j, k}\left(t_{1}\right)-W_{j, m}\left(t_{1}\right) W_{j, k}\left(t_{2}\right)\right\} \\
\quad+\sum_{m=u}^{i} \sum_{k=u}^{i} s_{k} s_{m}\left\{W_{j, m}\left(t_{2}\right) W_{j, k}\left(t_{1}\right)-W_{j, m}\left(t_{1}\right) W_{j, k}\left(t_{2}\right)\right\} \\
=\sum_{m=1}^{u-1} \sum_{k=u}^{i} s_{k} s_{m}\left\{W_{j, m}\left(t_{2}\right) W_{j, k}\left(t_{1}\right)-W_{j, m}\left(t_{1}\right) W_{j, k}\left(t_{2}\right)\right\} .
\end{aligned}
$$


Now, for $m<k$, we have

$$
\begin{aligned}
& W_{j, m}\left(t_{2}\right) W_{j, k}\left(t_{1}\right)-W_{j, m}\left(t_{1}\right) W_{j, k}\left(t_{2}\right) \\
&=\sum_{l_{1}=m}^{j-1} \sum_{l_{2}=k}^{j-1}\left(\begin{array}{l}
n \\
l_{1}
\end{array}\right)\left(\begin{array}{l}
n \\
l_{2}
\end{array}\right)\left(\phi\left(t_{2}\right)\right)^{l_{1}}\left(\phi\left(t_{1}\right)\right)^{l_{2}}-\sum_{l_{1}=m}^{j-1} \sum_{l_{2}=k}^{j-1}\left(\begin{array}{l}
n \\
l_{1}
\end{array}\right)\left(\begin{array}{l}
n \\
l_{2}
\end{array}\right)\left(\phi\left(t_{1}\right)\right)^{l_{1}}\left(\phi\left(t_{2}\right)\right)^{l_{2}} \\
&=\sum_{l_{1}=m}^{k-1} \sum_{l_{2}=k}^{j-1}\left(\begin{array}{l}
n \\
l_{1}
\end{array}\right)\left(\begin{array}{l}
n \\
l_{2}
\end{array}\right)\left(\phi\left(t_{2}\right)\right)^{l_{1}}\left(\phi\left(t_{1}\right)\right)^{l_{2}}-\sum_{l_{1}=m}^{k-1} \sum_{l_{2}=k}^{j-1}\left(\begin{array}{l}
n \\
l_{1}
\end{array}\right)\left(\begin{array}{l}
n \\
l_{2}
\end{array}\right)\left(\phi\left(t_{1}\right)\right)^{l_{1}}\left(\phi\left(t_{2}\right)\right)^{l_{2}} \\
& \\
& \quad+\sum_{l_{1}=k}^{j-1} \sum_{l_{2}=k}^{j-1}\left(\begin{array}{l}
n \\
l_{1}
\end{array}\right)\left(\begin{array}{l}
n \\
l_{2}
\end{array}\right)\left(\phi\left(t_{2}\right)\right)^{l_{1}}\left(\phi\left(t_{1}\right)\right)^{l_{2}}-\sum_{l_{1}=k}^{j-1} \sum_{l_{2}=k}^{j-1}\left(\begin{array}{l}
n \\
l_{1}
\end{array}\right)\left(\begin{array}{l}
n \\
l_{2}
\end{array}\right)\left(\phi\left(t_{1}\right)\right)^{l_{1}}\left(\phi\left(t_{2}\right)\right)^{l_{2}} \\
&=\sum^{k-1} \sum_{l_{1}=m}^{j-1}\left(\begin{array}{l}
n \\
l_{2}=k
\end{array}\right)\left(\begin{array}{l}
n \\
l_{1}
\end{array}\right)\left(\phi\left(t_{2}\right)\right)^{l_{1}}\left(\phi\left(t_{1}\right)\right)^{l_{1}}\left[\left(\phi\left(t_{1}\right)\right)^{l_{2}-l_{1}}-\left(\phi\left(t_{2}\right)\right)^{l_{2}-l_{1}}\right] \\
& \leq 0,
\end{aligned}
$$

where the last inequality holds since $\phi(t)$ is an increasing function of $t$. This completes the proof.

In what follows, we focus on the conditional probability

$$
\gamma_{j, k, n}(t, x)=\mathrm{P}\left(X_{j: n}-t>x \mid X_{k: n}<t<X_{j: n}\right),
$$

where $x, t>0$ and $1 \leq k<j \leq n$. It is clear that $\gamma_{j, k, n}(t, x)$ represents the residual lifetime of the live components in an $(n-k+1)$-out-of- $n$ system when the system has failed by time $t$ and $k$ of the components have failed. Note that $\gamma_{j, k, n}(t, x)$ can be represented as

$$
\begin{aligned}
\gamma_{j, k, n}(t, x) & =\frac{\mathrm{P}\left(X_{j: n}-t>x, X_{k: n}<t<X_{j: n}\right)}{\mathrm{P}\left(X_{k: n}<t<X_{j: n}\right)} \\
& =\frac{\sum_{l=k}^{j-1} \mathrm{P}\left(X_{j: n}-t>x, X_{l: n}<t<X_{l+1: n}\right)}{\mathrm{P}\left(X_{k: n}<t<X_{j: n}\right)} \\
& =\sum_{l=k}^{j-1} B_{j, l, n}(t, x) K_{l, j, k}^{n}(t),
\end{aligned}
$$

where $B_{j, l, n}(t, x)=\mathrm{P}\left(X_{j: n}-t>x \mid X_{l: n}<t<X_{l+1: n}\right)$ and

$$
\begin{aligned}
K_{l, j, k}^{n}(t) & =\frac{\mathrm{P}\left(X_{l: n}<t<X_{l+1: n}\right)}{\mathrm{P}\left(X_{k: n}<t<X_{j: n}\right)} \\
& =\frac{\left(\begin{array}{c}
n \\
l
\end{array}\right)(F(t))^{l}(1-F(t))^{n-l}}{\sum_{m=k}^{j-1}\left(\begin{array}{l}
n \\
m
\end{array}\right)(F(t))^{m}(1-F(t))^{n-m}} \\
& =\frac{\left(\begin{array}{c}
n \\
l
\end{array}\right)(\phi(t))^{l}}{\sum_{m=k}^{j-1}\left(\begin{array}{l}
n \\
m
\end{array}\right)(\phi(t))^{m}}, \quad 1 \leq k \leq l<j \leq n .
\end{aligned}
$$

Remark 2.1. The above results readily yield the following representation for $P_{j, T}(t, x)$ :

$$
P_{j, T}(t, x)=\sum_{k=1}^{i} p_{k}(t) \gamma_{j, k, n}(t, x)=\sum_{k=1}^{i} p_{k}(t) \sum_{l=k}^{j-1} B_{j, l, n}(t, x) K_{l, j, k}^{n}(t) .
$$


Lemma 2.2. For $n \geq j>l$, we have

$$
\left(X_{j: n}-t \mid X_{l: n}<t<X_{l+1: n}\right) \stackrel{\mathrm{D}}{=} X_{j-l: n-l}^{t},
$$

where $X_{j-l: n-l}^{t}$ denotes the $(j-l)$ th order statistic among $n-l$ i.i.d. random variables with a left-truncated distribution with survival function $\bar{F}(x \mid t)=\bar{F}(t+x) / \bar{F}(t)$ for $x, t>0$.

Proof. We have

$$
\begin{aligned}
B_{j, l, n}(t, x)= & \frac{\mathrm{P}\left(X_{j: n}>t+x, X_{l: n}<t<X_{l+1: n}\right)}{\mathrm{P}\left(X_{l: n}<t<X_{l+1: n}\right)} \\
= & \left(\sum_{m=n-j+1}^{n-l} \frac{n !}{l !(n-l-m) ! m !} F^{l}(t)[F(x+t)-F(t)]^{n-l-m}[1-F(x+t)]^{m}\right) \\
& \times\left(\left(\begin{array}{c}
n \\
l
\end{array}\right)(F(t))^{l}(1-F(t))^{n-l}\right)^{-1} \\
= & \sum_{m=n-j+1}^{n-l}\left(\begin{array}{c}
n-l \\
m
\end{array}\right)(\bar{F}(x \mid t))^{m}(1-\bar{F}(x \mid t))^{n-l-m},
\end{aligned}
$$

where $\bar{F}(x \mid t)=\bar{F}(x+t) / \bar{F}(t)$. This establishes the required distributional result.

Remark 2.2. It should also be noted that

$$
\left(X_{j: n}-t \mid X_{l: n}<t<X_{l+1: n}\right) \stackrel{\mathrm{D}}{=}\left(X_{j-l: n-l}-t \mid X_{1: n-l}>t\right),
$$

since

$$
\begin{aligned}
\mathrm{P}\left(X_{j-l: n-l}-t>x \mid X_{1: n-l}>t\right) & =\frac{\mathrm{P}\left(X_{j-l: n-l}-t>x, X_{1: n-l}>t\right)}{\mathrm{P}\left(X_{1: n-l}>t\right)} \\
& =\frac{\sum_{m=n-j+1}^{n-l}\left(\begin{array}{c}
n-l \\
m
\end{array}\right)[F(x+t)-F(t)]^{n-l-m}[\bar{F}(x+t)]^{m}}{(\bar{F}(t))^{n-l}} \\
& =\sum_{m=n-j+1}^{n-l}\left(\begin{array}{c}
n-l \\
m
\end{array}\right)\left\{\frac{\bar{F}(x+t)}{\bar{F}(t)}\right\}^{m}\left\{1-\frac{\bar{F}(t+x)}{\bar{F}(t)}\right\}^{n-l-m} \\
& =\sum_{m=n-j+1}^{n-l}\left(\begin{array}{c}
n-l \\
m
\end{array}\right)(\bar{F}(x \mid t))^{m}(1-\bar{F}(x \mid t))^{n-l-m}
\end{aligned}
$$

Lemma 2.3. Under the assumption that the underlying distribution function is absolutely continuous, for $j>l \geq m$, we have

$$
\left(X_{j: n}-t \mid X_{l: n}<t<X_{l+1: n}\right) \leq \operatorname{lr}\left(X_{j: n}-t \mid X_{m: n}<t<X_{m+1: n}\right) .
$$

Proof. It is well known (see Theorem 1.C.37 and Corollaries 1.C.38 and 1.C.39 of [23]) that

$$
X_{k-1: m-1} \leq \operatorname{lr} X_{k: m} \quad \text { for } k=2,3, \ldots, m
$$

and

$$
X_{k: m-1} \geq \operatorname{lr} X_{k: m} \quad \text { for } k=1,2, \ldots, m-1 .
$$


Hence, we have

$$
X_{j-l: n-l}^{t} \leq_{\mathrm{lr}} X_{j+1-l: n-l+1}^{t}={ }_{\mathrm{lr}} X_{j-(l-1): n-(l-1)}^{t},
$$

which means that

$$
\left(X_{j: n}-t \mid X_{l: n}<t<X_{l+1: n}\right) \leq_{\text {lr }}\left(X_{j: n}-t \mid X_{l-1: n}<t<X_{l: n}\right),
$$

as required.

Lemma 2.4. If $F$ is absolutely continuous then, for $k \leq m$, we have

$$
\left(X_{j: n}-t \mid X_{k: n}<t<X_{j: n}\right) \geq \operatorname{lr}\left(X_{j: n}-t \mid X_{m: n}<t<X_{j: n}\right) .
$$

Proof. Let us denote the densities of $\left(X_{j: n}-t \mid X_{k: n}<t<X_{j: n}\right)$ and $\left(X_{j: n}-t \mid X_{l: n}<\right.$ $\left.t<X_{l+1: n}\right)$ by $f_{t}^{j, k, n}(x)$ and $f_{j, l, n, t}(x)$, respectively. Then, we have

$$
f_{t}^{j, k, n}(x)=\sum_{l=j}^{k-1} f_{j, l, n, t}(x) K_{l, j, k}^{n}(t) .
$$

To prove the claimed result, we need to show that the function

$$
\frac{f_{t}^{j, k, n}(x)}{f_{t}^{j, k+1, n}(x)}
$$

is increasing in $x$, that is, we need to show that, for $x_{1}<x_{2}$,

$$
\frac{\sum_{l=k}^{j-1} f_{j, l, n, t}\left(x_{1}\right) K_{l, j, k}^{n}(t)}{\sum_{l=k+1}^{j-1} f_{j, l, n, t}\left(x_{1}\right) K_{l, j, k+1}^{n}(t)} \leq \frac{\sum_{l=k}^{j-1} f_{j, l, n, t}\left(x_{2}\right) K_{l, j, k}^{n}(t)}{\sum_{l=k+1}^{j-1} f_{j, l, n, t}\left(x_{2}\right) K_{l, j, k+1}^{n}(t)}
$$

This is equivalent to showing, by using (2.4), that

$$
\frac{\sum_{l=k}^{j-1} f_{j, l, n, t}\left(x_{1}\right)\left(\begin{array}{l}
n \\
l
\end{array}\right)(\phi(t))^{l}}{\sum_{m=k+1}^{j-1} f_{j, m, n, t}\left(x_{1}\right)\left(\begin{array}{l}
n \\
m
\end{array}\right)(\phi(t))^{m}} \leq \frac{\sum_{l=k}^{j-1} f_{j, l, n, t}\left(x_{2}\right)\left(\begin{array}{l}
n \\
l
\end{array}\right)(\phi(t))^{l}}{\sum_{m=k+1}^{j-1} f_{j, m, n, t}\left(x_{2}\right)\left(\begin{array}{l}
n \\
m
\end{array}\right)(\phi(t))^{m}}
$$

or

$$
\sum_{l=k}^{j-1} \sum_{m=k+1}^{j-1}\left(\begin{array}{l}
n \\
l
\end{array}\right)\left(\begin{array}{l}
n \\
m
\end{array}\right)(\phi(t))^{l+m}\left\{f_{j, l, n, t}\left(x_{1}\right) f_{j, m, n, t}\left(x_{2}\right)-f_{j, l, n, t}\left(x_{2}\right) f_{j, m, n, t}\left(x_{1}\right)\right\} \leq 0 .
$$

We can easily verify that this inequality holds if and only if the following inequality holds:

$$
\sum_{m=k+1}^{j-1}\left(\begin{array}{l}
n \\
k
\end{array}\right)\left(\begin{array}{l}
n \\
m
\end{array}\right)(\phi(t))^{k+m}\left\{f_{j, k, n, t}\left(x_{1}\right) f_{j, m, n, t}\left(x_{2}\right)-f_{j, k, n, t}\left(x_{2}\right) f_{j, m, n, t}\left(x_{1}\right)\right\} \leq 0 .
$$

However, for $m>k$, we know from Lemma 2.3 that $\left(X_{j: n}-t \mid X_{k: n}<t<X_{k+1: n}\right) \geq_{\text {lr }}$ $\left(X_{j: n}-t \mid X_{m: n}<t<X_{m+1: n}\right)$, which is equivalent to

$$
f_{j, k, n, t}\left(x_{1}\right) f_{j, m, n, t}\left(x_{2}\right)-f_{j, k, n, t}\left(x_{2}\right) f_{j, m, n, t}\left(x_{1}\right) \leq 0 .
$$

This leads to the inequality in (2.6), completing the proof. 
We now present the main result of this section.

Theorem 2.3. Let $\boldsymbol{p}_{1}(t)$ and $\boldsymbol{p}_{2}(t)$ for a fixed $t>0$ be the vectors of coefficients in representation (2.5) of two coherent systems of order $n$, both based on components with i.i.d. lifetimes having a common continuous distribution function $F$. Denote by $T_{1}$ and $T_{2}$ the respective lifetimes of the two systems with signatures of type (A). Then, for $j>i$, we have the following orderings.

(a) If $\boldsymbol{p}_{1}(t) \leq_{\mathrm{st}} \boldsymbol{p}_{2}(t)$ then $\left(X_{j: n}-t \mid T_{1}<t<X_{j: n}\right) \geq_{\mathrm{st}}\left(X_{j: n}-t \mid T_{2}<t<X_{j: n}\right)$.

(b) If $\boldsymbol{p}_{1}(t) \leq \mathrm{hr} \boldsymbol{p}_{2}(t)$ then $\left(X_{j: n}-t \mid T_{1}<t<X_{j: n}\right) \geq_{\mathrm{rh}}\left(X_{j: n}-t \mid T_{2}<t<X_{j: n}\right)$.

(c) If $\boldsymbol{p}_{1}(t) \leq_{\operatorname{lr}} \boldsymbol{p}_{2}(t)$ then $\left(X_{j: n}-t \mid T_{1}<t<X_{j: n}\right) \geq_{\operatorname{lr}}\left(X_{j: n}-t \mid T_{2}<t<X_{j: n}\right)$, when the parent distribution $F$ is absolutely continuous.

Proof. Orderings (a)-(c) follow readily from the above mixture representations, Lemma 2.4, and properties of mixtures given in [23].

\subsection{Ageing properties of the live components}

In this subsection we examine some ageing properties of $P_{j, T}(t, x)$. We show that when the components of the system are IFR (increasing failure rate), then $P_{j, T}(t, x)$ is a decreasing function of time. To this end, we need the following lemmas.

Lemma 2.5. If $h(t)=f(t) / \bar{F}(t)$ is increasing (i.e. $F$ is IFR) then

$$
H_{n}^{j}(t, x)=\mathrm{P}\left(X_{j: n}-t>x \mid X_{1: n}>t\right) \quad \text { for } j \geq 1
$$

is decreasing in $t$ for all $x \geq 0$.

Proof. Note that the random variable $\left(X_{j: n}-t \mid X_{1: n}>t\right)$ can be considered as the $j$ th order statistic in a random sample of size $n$ from a left-truncated distribution with survival function $\bar{F}(t+x) / \bar{F}(t)$ for $x, t>0$. Hence, the survival function $H_{n}^{j}(t, x)$ is given by

$$
\begin{aligned}
H_{n}^{j}(t, x) & =\sum_{m=0}^{j-1}\left(\begin{array}{l}
n \\
m
\end{array}\right)\left\{1-\frac{\bar{F}(t+x)}{\bar{F}(t)}\right\}^{m}\left\{\frac{\bar{F}(t+x)}{\bar{F}(t)}\right\}^{n-m} \\
& =\int_{1-\bar{F}(t+x) / \bar{F}(t)}^{1} \frac{n !}{(j-1) !(n-j) !} t^{j-1}(1-t)^{n-j} \mathrm{~d} t .
\end{aligned}
$$

Since $h(t)$ is increasing if and only if $\bar{F}(t+x) / \bar{F}(t)$ is decreasing in $t$, from (2.7) we see that $H_{n}^{j}(t, x)$ is a decreasing function of $t$.

Lemma 2.6. Let $h(t)$, the hazard rate of the components of the system, be increasing in $t$ for $t>0$. Then $\gamma_{j, k, n}(t, x)$ is a decreasing function of $t$.

Proof. Note that

$$
\frac{\mathrm{d}}{\mathrm{d} t} \gamma_{j, k, n}(t, x)=\sum_{l=k}^{j-1}\left(\frac{\mathrm{d}}{\mathrm{d} t} H_{n-l}^{j-l}(t, x)\right) K_{l, j, k}^{n}(t)+\sum_{l=k}^{j-1} H_{n-l}^{j-l}(t, x)\left(\frac{\mathrm{d}}{\mathrm{d} t} K_{l, j, k}^{n}(t)\right) .
$$


The first term on the right-hand side is negative by the assumption of the lemma and Lemma 2.5. Hence, we just need to prove that the second term is negative. On taking $U_{m}(t)=\left(\begin{array}{l}n \\ m\end{array}\right) t^{m}$, we have

$$
\begin{aligned}
& \sum_{l=k}^{j-1} H_{n-l}^{j-l}(t, x)\left(\frac{\mathrm{d}}{\mathrm{d} t} K_{l, j, k}^{n}(t)\right) \\
& \quad=\frac{\sum_{l=k}^{j-1} H_{n-l}^{j-l}(t, x)\left\{U_{l}^{\prime}(t) \sum_{m=k}^{j-1} U_{m}(t)-U_{l}(t) \sum_{m=k}^{j-1} U_{m}^{\prime}(t)\right\}}{\left\{\sum_{m=k}^{j-1} U_{m}(t)\right\}^{2}} .
\end{aligned}
$$

After some algebraic manipulations, it can be shown that the numerator of the above expression can be written as

$$
\begin{aligned}
\sum_{l=k}^{j-1} \sum_{m=k}^{j-1} U_{l}^{\prime}(t) U_{m}(t)\left\{H_{n-l}^{j-l}(t, x)-H_{n-m}^{j-m}(t, x)\right\} \\
=\sum_{l=k}^{j-1} \sum_{m=k}^{l} U_{l}^{\prime}(t) U_{m}(t)\left\{H_{n-l}^{j-l}(t)-H_{n-m}^{j-m}(t)\right\} \\
\quad+\sum_{m=k}^{j-1} \sum_{l=k}^{m} U_{l}^{\prime}(t) U_{m}(t)\left\{H_{n-l}^{j-l}(t)-H_{n-m}^{j-m}(t)\right\} \\
=\sum_{l=j}^{k-1} \sum_{m=j}^{l}\left\{U_{l}^{\prime}(t) U_{m}(t)-U_{m}^{\prime}(t) U_{l}(t)\right\}\left\{H_{n-l}^{j-l}(t, x)-H_{n-m}^{j-m}(t, x)\right\} \\
=\sum_{l=j}^{k-1} \sum_{m=j}^{l}(l-m)\left\{\left(\begin{array}{c}
n \\
l
\end{array}\right)\left(\begin{array}{c}
n \\
m
\end{array}\right) t^{l+m-1}\right\}\left\{H_{n-l}^{j-l}(t, x)-H_{n-m}^{j-m}(t, x)\right\} \\
\geq 0,
\end{aligned}
$$

where the last inequality follows from the fact that, for $j>l \geq m$, we have

$$
\left(X_{j: n}-t \mid X_{l: n}<t<X_{l+1: n}\right) \leq_{\operatorname{lr}}\left(X_{j: n}-t \mid X_{m: n}<t<X_{m+1: n}\right),
$$

and, hence, $H_{n-l}^{j-l}(t, x) \leq H_{n-m}^{j-m}(t, x)$. This completes the proof.

Now we present the main result of this subsection.

Theorem 2.4. Let $h(t)$, the hazard rate of the components of the system, be increasing in $t$ for $t>0$. Then $P_{j, T}(t, x)$ is a decreasing function of $t$.

Proof. Under the assumption that $P_{j, T}(t, x)$ is differentiable in terms of $t$, we have

$$
\frac{\mathrm{d}}{\mathrm{d} t} P_{j, T}(t, x)=\sum_{k=1}^{i}\left(\frac{\mathrm{d}}{\mathrm{d} t} \gamma_{j, k, n}(t, x)\right) p_{k}(t)+\sum_{k=1}^{i} \gamma_{j, k, n}(t, x)\left(\frac{\mathrm{d}}{\mathrm{d} t} p_{k}(t)\right) .
$$

Based on the assumption of the theorem and the result in Lemma 2.6, the first term on the right-hand side is negative and so it is sufficient to show that the second term is also negative. 
We have

$$
\begin{aligned}
& \sum_{k=1}^{i} \gamma_{j, k, n}(t, x)\left(\frac{\mathrm{d}}{\mathrm{d} t} p_{k}(t)\right) \\
& \quad=\frac{\sum_{k=1}^{i} \gamma_{j, k, n}(t, x)\left\{s_{k} W_{j, k}^{\prime}(t) \sum_{m=1}^{i} s_{m} W_{j, m}(t)-s_{k} W_{j, k}(t) \sum_{m=1}^{i} s_{m} W_{j, m}^{\prime}(t)\right\}}{\left\{\sum_{m=1}^{i} s_{m} W_{j, m}(t)\right\}^{2}} .
\end{aligned}
$$

After some algebraic manipulations, we can show that the numerator of the above expression can be rewritten as

$$
\begin{aligned}
& \sum_{k=1}^{i} \sum_{m=1}^{i} s_{k} s_{m} \gamma_{j, k, n}(t, x)\left\{W_{j, k}^{\prime}(t) W_{j, m}(t)-W_{j, k}(t) W_{j, m}^{\prime}(t)\right\} \\
& \quad=\sum_{k=1}^{i} \sum_{m=1}^{k} s_{k} s_{m}\left\{W_{j, k}^{\prime}(t) W_{j, m}(t)-W_{j, k}(t) W_{j, m}^{\prime}(t)\right\}\left\{\gamma_{j, k, n}(t, x)-\gamma_{j, m, n}(t, x)\right\} .
\end{aligned}
$$

Since $\left(X_{j: n}-t \mid X_{k: n}<t<X_{j: n}\right) \geq_{\text {st }}\left(X_{j: n}-t \mid X_{k+1: n}<t<X_{j: n}\right)$, we have, for $m \leq k, \gamma_{j, k, n}(t, x)-\gamma_{j, m, n}(t, x) \leq 0$. On the other hand,

$$
\begin{aligned}
& W_{j, k}^{\prime}(t) W_{j, m}(t)-W_{j, k}(t) W_{j, m}^{\prime}(t) \\
& =\left\{\sum_{l=k}^{j-1}\left(\begin{array}{l}
n \\
l
\end{array}\right) \phi^{l}(t)\right\}^{\prime}\left\{\sum_{l=m}^{j-1}\left(\begin{array}{l}
n \\
l
\end{array}\right) \phi^{l}(t)\right\}-\left\{\sum_{l=k}^{j-1}\left(\begin{array}{l}
n \\
l
\end{array}\right) \phi^{l}(t)\right\}\left\{\sum_{l=m}^{j-1}\left(\begin{array}{l}
n \\
l
\end{array}\right) \phi^{l}(t)\right\}^{\prime} \\
& =\phi^{\prime}(t) \sum_{l_{1}=k}^{j-1} \sum_{l_{2}=m}^{j-1} l_{1}\left(\begin{array}{l}
n \\
l_{1}
\end{array}\right)\left(\begin{array}{l}
n \\
l_{2}
\end{array}\right) \phi^{l_{1}+l_{2}-1}(t)-\phi^{\prime}(t) \sum_{l_{1}=k}^{j-1} \sum_{l_{2}=m}^{j-1} l_{2}\left(\begin{array}{l}
n \\
l_{1}
\end{array}\right)\left(\begin{array}{l}
n \\
l_{2}
\end{array}\right) \phi^{l_{1}+l_{2}-1}(t) \\
& =\phi^{\prime}(t) \sum_{l_{1}=k}^{j-1} \sum_{l_{2}=m}^{k-1}\left(\begin{array}{l}
n \\
l_{1}
\end{array}\right)\left(\begin{array}{l}
n \\
l_{2}
\end{array}\right) \phi^{l_{1}+l_{2}-1}\left(l_{1}-l_{2}\right)(t) \\
& \geq 0 .
\end{aligned}
$$

Hence, the expression on the right-hand side of (2.9) is negative, and, consequently, $P_{j, T}(t, x)$ is decreasing in $t$, which completes the proof.

\subsection{Stochastic comparisons between the live components}

In what follows, we consider two coherent systems having the same structure with i.i.d. components distributed according to common distribution functions $F$ and $G$, respectively. Let $T_{1}$ and $T_{2}$ be the corresponding lifetimes of the two systems. We show that, under the condition that the hazard rate of the components with system lifetime $T_{1}$ is less than the hazard rate of the components with system lifetime $T_{2}$, the live components of the system with lifetime $T_{1}$ are more reliable than the live components of the system with lifetime $T_{2}$. For this specific purpose, we need the following lemmas.

Lemma 2.7. We have $X \leq_{\mathrm{hr}} Y$ if and only if

$$
\left(X_{j: n}-t \mid X_{l: n}<t<X_{l+1: n}\right) \leq_{\mathrm{st}}\left(Y_{j: n}-t \mid Y_{l: n}<t<Y_{l+1: n}\right) .
$$


Proof. Let $B_{j, l, n}^{X}(t, x)$ be the survival function of $\mathrm{P}\left(X_{j: n}-t>x \mid X_{l: n}<t<X_{l+1: n}\right)$. Then from Lemma 2.2 we have

$$
\begin{aligned}
B_{j, l, n}^{X}(t, x) & =\sum_{m=n-j+1}^{n-l}\left(\begin{array}{c}
n-l \\
m
\end{array}\right)\left(1-F_{t}(x)\right)^{m}\left(F_{t}(x)\right)^{n-l-m} \\
& =1-\sum_{m=0}^{n-j}\left(\begin{array}{c}
n-l \\
m
\end{array}\right)\left(1-F_{t}(x)\right)^{m}\left(F_{t}(x)\right)^{n-l-m} \\
& =1-j\left(\begin{array}{c}
l \\
j
\end{array}\right) \int_{\bar{F}(t+x) / \bar{F}(t)}^{1} u^{j-1}(1-u)^{j-l} \mathrm{~d} u .
\end{aligned}
$$

However, the assumption that $X \leq_{\mathrm{hr}} Y$ implies that $\bar{F}(t+x) / \bar{F}(t) \leq \bar{G}(t+x) / \overline{G(t)}$. Hence, $B_{j, l, n}^{X}(t, x) \leq B_{j, l, n}^{Y}(t, x)$, where $B_{j, l, n}^{Y}(t, x)$ is the reliability function of $\left(Y_{j: n}-t \mid Y_{l: n}<t<\right.$ $\left.Y_{l+1: n}\right)$. This leads to the stochastic ordering in (2.10), completing the proof.

Lemma 2.8. Let $X \leq_{\mathrm{hr}} Y$. Then

$$
\left(X_{j: n}-t \mid X_{k: n}<t<X_{j: n}\right) \leq_{\mathrm{st}}\left(Y_{j: n}-t \mid Y_{k: n}<t<Y_{j: n}\right) .
$$

Proof. We have

$$
\begin{aligned}
\gamma_{j, k, n}^{X}(t, x)-\gamma_{j, k, n}^{Y}(t, x)= & \sum_{l=k}^{j-1} B_{j, l, n}^{X}(t, x) K_{l, j, k}^{n, X}(t)-\sum_{l=k}^{j-1} B_{j, l, n}^{Y}(t, x) K_{l, j, k}^{n, Y}(t) \\
= & \frac{\sum_{l=k}^{j-1} B_{j, l, n}^{X}(t, x)\left(\begin{array}{l}
n \\
l
\end{array}\right)\left(\phi_{X}(t)\right)^{l}}{\sum_{m=k}^{j-1}\left(\begin{array}{l}
n \\
m
\end{array}\right)\left(\phi_{X}(t)\right)^{m}}-\frac{\sum_{l=k}^{j-1} B_{j, l, n}^{Y}(t, x)\left(\begin{array}{l}
n \\
l
\end{array}\right)\left(\phi_{Y}(t)\right)^{l}}{\sum_{m=k}^{j-1}\left(\begin{array}{l}
n \\
m
\end{array}\right)\left(\phi_{Y}(t)\right)^{m}} \\
= & \frac{\left\{\sum_{l=k}^{j-1} B_{j, l, n}^{X}(t, x)\left(\begin{array}{l}
n \\
l
\end{array}\right)\left(\phi_{X}(t)\right)^{l}\right\}\left\{\sum_{m=k}^{j-1}\left(\begin{array}{l}
n \\
m
\end{array}\right)\left(\phi_{Y}(t)\right)^{m}\right\}}{\left\{\sum_{m=k}^{k-1}\left(\begin{array}{l}
n \\
m
\end{array}\right)\left(\phi_{Y}(t)\right)^{m}\right\}\left\{\sum_{m=k}^{j-1}\left(\begin{array}{c}
n \\
m
\end{array}\right)\left(\phi_{X}(t)\right)^{m}\right\}} \\
& -\frac{\left\{\sum_{l=k}^{j-1} B_{j, l, t}^{Y}(t, x)\left(\begin{array}{l}
n \\
l
\end{array}\right)\left(\phi_{Y}(t)\right)^{l}\right\}\left\{\sum_{m=k}^{j-1}\left(\begin{array}{c}
n \\
m
\end{array}\right)\left(\phi_{X}(t)\right)^{m}\right\}}{\left\{\sum_{m=k}^{k-1}\left(\begin{array}{l}
n \\
m
\end{array}\right)\left(\phi_{Y}(t)\right)^{m}\right\}\left\{\sum_{m=k}^{j-1}\left(\begin{array}{c}
n \\
m
\end{array}\right)\left(\phi_{X}(t)\right)^{m}\right\}}
\end{aligned}
$$

The numerator of the above expression can be rewritten as

$$
\begin{aligned}
\sum_{m=k}^{j-1}\left(\begin{array}{l}
n \\
m
\end{array}\right) \sum_{l=k}^{j-1}\left(\begin{array}{l}
n \\
l
\end{array}\right)\left\{\left(\phi_{X}(t)\right)^{l}\left(\phi_{Y}(t)\right)^{m} B_{j, l, n}^{X}(t, x)-\left(\phi_{X}(t)\right)^{m}\left(\phi_{Y}(t)\right)^{l} B_{j, l, n}^{Y}(t, x)\right\} \\
=\sum_{m=k}^{j-1}\left(\begin{array}{l}
n \\
m
\end{array}\right) \sum_{l=k}^{j-1}\left(\begin{array}{l}
n \\
l
\end{array}\right)\left(\phi_{X}(t)\right)^{l}\left(\phi_{Y}(t)\right)^{m}\left\{B_{j, l, n}^{X}(t, x)-B_{j, l, n}^{Y}(t, x)\right\} \\
\quad+\sum_{m=k}^{j-1}\left(\begin{array}{l}
n \\
m
\end{array}\right) \sum_{l=k}^{j-1}\left(\begin{array}{l}
n \\
l
\end{array}\right)\left\{\left(\phi_{X}(t)\right)^{l}\left(\phi_{Y}(t)\right)^{m}-\left(\phi_{X}(t)\right)^{m}\left(\phi_{Y}(t)\right)^{l}\right\} B_{j, l, n}^{Y}(t, x) .
\end{aligned}
$$

The assumption that $X \leq_{\mathrm{hr}} Y$ implies, from Lemma 2.7, that $B_{j, l, n}^{X}(t, x)-B_{j, l, n}^{Y}(t, x) \leq 0$. Hence, the first term on the right-hand side in the above expression is negative. Next, after 
performing some algebraic manipulations, it can be shown that the second term can be expressed as

$$
\begin{gathered}
\sum_{m=k}^{j-1}\left(\begin{array}{l}
n \\
m
\end{array}\right) \sum_{l=k}^{m}\left(\begin{array}{l}
n \\
l
\end{array}\right)\left\{\left(\phi_{X}(t)\right)^{l}\left(\phi_{Y}(t)\right)^{m}-\left(\phi_{X}(t)\right)^{m}\left(\phi_{Y}(t)\right)^{l}\right\} B_{j, l, n}^{Y}(t, x) \\
+\sum_{m=k}^{j-1}\left(\begin{array}{l}
n \\
m
\end{array}\right) \sum_{l=m}^{j-1}\left(\begin{array}{l}
n \\
l
\end{array}\right)\left\{\left(\phi_{X}(t)\right)^{l}\left(\phi_{Y}(t)\right)^{m}-\left(\phi_{X}(t)\right)^{m}\left(\phi_{Y}(t)\right)^{l}\right\} B_{j, l, n}^{Y}(t, x) \\
=\sum_{m=k}^{j-1}\left(\begin{array}{l}
n \\
m
\end{array}\right) \sum_{l=k}^{m}\left(\begin{array}{l}
n \\
l
\end{array}\right)\left(\phi_{X}(t)\right)^{m}\left(\phi_{Y}(t)\right)^{m}\left\{\left(\phi_{X}(t)\right)^{l-m}-\left(\phi_{Y}(t)\right)^{l-m}\right\} \\
\times\left\{B_{j, l, n}^{Y}(t, x)-\bar{B}_{j, m, t}^{Y}(x)\right\} .
\end{gathered}
$$

Now, since $B_{j, l, t}^{Y}(t, x)-B_{j, m, t}^{Y}(t, x) \geq 0$ and $\left(\phi_{X}(t)\right)^{l-m}-\left(\phi_{Y}(t)\right)^{l-m} \leq 0$, the second term on the right-hand side of (2.11) is also negative. This completes the proof.

The following theorem is the main result of this subsection.

Theorem 2.5. Let $X \leq{ }_{\mathrm{hr}} Y$, and let $T_{1}$ and $T_{2}$ denote the lifetimes of two systems with signatures of type (A). Then, for $j>i,\left(X_{j: n}-t \mid T_{1}<t<X_{j: n}\right) \leq_{\mathrm{st}}\left(Y_{j: n}-t \mid T_{2}<t<Y_{j: n}\right)$.

Proof. Note that

$$
\begin{aligned}
& \mathrm{P}\left(X_{j: n}-t>x \mid T_{1}<t<X_{j: n}\right)=\sum_{k=1}^{i} p_{k}^{X}(t) \gamma_{j, k, n}^{X}(t, x), \\
& \mathrm{P}\left(Y_{j: n}-t>x \mid T_{2}<t<Y_{j: n}\right)=\sum_{k=1}^{i} p_{k}^{Y}(t) \gamma_{j, k, n}^{Y}(t, x),
\end{aligned}
$$

where

$$
p_{k}^{X}(t)=\frac{s_{k} W_{j, k}^{X}(t)}{\sum_{m=1}^{i} s_{m} W_{j, m}^{X}(t)}, \quad p_{k}^{Y}(t)=\frac{s_{k} W_{j, k}^{Y}(t)}{\sum_{m=1}^{i} s_{m} W_{j, m}^{Y}(t)} .
$$

To prove the required result, we need to show that

$$
\begin{aligned}
& \sum_{k=1}^{i} p_{k}^{X}(t) \gamma_{j, k, n}^{X}(t, x)-\sum_{k=1}^{i} p_{k}^{Y}(t) \gamma_{j, k, n}^{Y}(t, x) \\
& \quad=\sum_{k=1}^{i} \frac{s_{k} W_{j, k}^{X}(t)}{\sum_{m=1}^{i} s_{m} W_{j, m}^{X}(t)} \gamma_{j, k, n}^{X}(t, x)-\sum_{k=1}^{i} \frac{s_{k} W_{j, k}^{Y}(t)}{\sum_{m=1}^{i} s_{m} W_{j, m}^{Y}(t)} \gamma_{j, k, n}^{Y}(t, x) \\
& \quad \leq 0 .
\end{aligned}
$$

This, in turn, is equivalent to showing that

$$
\sum_{k=1}^{i} \sum_{m=1}^{i} s_{m} s_{k} W_{j, k}^{X}(t) W_{j, m}^{Y}(t) \gamma_{j, k, n}^{X}(t, x)-\sum_{k=1}^{i} \sum_{m=1}^{i} s_{m} s_{k} W_{j, m}^{X}(t) W_{j, k}^{Y}(t) \gamma_{j, k, n}^{Y}(t, x) \leq 0
$$


The left-hand side of the above inequality can be written as

$$
\sum_{k=1}^{i} \sum_{m=k}^{i} s_{k} s_{m}\left\{W_{j, k}^{X}(t) W_{j, m}^{Y}(t)-W_{j, m}^{X}(t) W_{j, k}^{Y}(t)\right\}\left\{\gamma_{j, k, n}^{X}(t, x)-\gamma_{j, k, n}^{X}(t, x)\right\} .
$$

From Lemma 2.8, for $m \geq k$, we have $\gamma_{j, k, n}^{Y}(t, x) \leq \gamma_{j, k, n}^{X}(t, x)$. Moreover, it is not difficult to show that

$$
W_{j, k}^{X}(t) W_{j, m}^{Y}(t)-W_{j, m}^{X}(t) W_{j, k}^{Y}(t) \leq 0 .
$$

So, the expression in (2.12) is negative, which completes the proof.

\section{Inactivity times of the failed components}

In this section we study the properties of inactivity times of the components of a coherent system that have failed in the system, but the system is still functioning at time $t$. Let $T$ be the lifetime of a coherent system of order $n$, and denote by $X_{1}, X_{2}, \ldots, X_{n}$ the i.i.d. lifetimes of its components with the underlying distribution of the components being $F$. In what follows, we suppose that the signature of the system has the form

$$
s=\left(0, \ldots, 0, s_{i}, s_{i+1}, \ldots, s_{n}\right), \quad 1<i \leq n .
$$

Examples of coherent systems of order 4 with signature vector of the form (3.1) are presented in Table 2 .

In such coherent systems, we have

$$
\mathrm{P}\left(T=X_{k: n}\right)=0 \quad \text { for } k=1,2, \ldots, i .
$$

That is, a component with lifetime $X_{k: n}, k=1,2, \ldots, i$, cannot cause the failure of the system and, hence, when the system is working at time $t$, i.e. $T>t$, it is possible for such components to have already failed in the system, i.e. $X_{k: n}<t$. Thus, the following conditional random variable will be of interest for reliability and system designers:

$$
\left(t-X_{k: n} \mid T>t, X_{k: n}<t\right) .
$$

This conditional random variable is in fact the inactivity time of the component with lifetime $X_{k: n}$ when the system is working at time $t$ and the component with lifetime $X_{k: n}$ has failed

TABLE 2: Coherent systems with four components and signatures of the form (3.1).

\begin{tabular}{cc}
\hline$T=T\left(X_{1}, X_{2}, X_{3}, X_{4}\right)$ & Signature \\
\hline$X_{2: 2}=\max \left(X_{1}, X_{2}\right)$ (2-parallel) & $\left(0, \frac{1}{6}, \frac{1}{3}, \frac{1}{2}\right)$ \\
$\max \left(X_{2}, \min \left(X_{1}, X_{3}\right)\right)($ consecutive 2-out-of-3: $F)$ & $\left(0, \frac{1}{3}, \frac{5}{12}, \frac{1}{4}\right)$ \\
$\max \left(X_{1}, \min \left(X_{2}, X_{3}, X_{4}\right)\right)$ & $\left(0, \frac{1}{2}, \frac{1}{4}, \frac{1}{4}\right)$ \\
$\max \left(X_{1}, \min \left(X_{2}, X_{3}\right), \min \left(X_{3}, X_{4}\right)\right)$ & $\left(0, \frac{1}{6}, \frac{7}{12}, \frac{1}{4}\right)$ \\
$X_{3: 3}=\max \left(X_{1}, X_{2}, X_{3}\right)(3$-parallel) & $\left(0,0, \frac{1}{4}, \frac{3}{4}\right)$ \\
$\max \left(X_{2: 3}, X_{4}\right)$ & $\left(0,0, \frac{3}{4}, \frac{1}{4}\right)$ \\
$\min \left(\max \left(X_{1}, X_{2}, X_{3}\right), \max \left(X_{2}, X_{3}, X_{4}\right)\right)($ consecutive 3-out-of-4: $F)$ & $\left(0,0, \frac{1}{2}, \frac{1}{2}\right)$ \\
$X_{4: 4}=\max \left(X_{1}, X_{2}, X_{3}, X_{4}\right)$ (parallel) & $(0,0,0,1)$ \\
\hline
\end{tabular}


sometime before $t, k=1, \ldots, i$. In the sequel, we are interested in examining the ageing and stochastic properties of the conditional probability

$$
P_{j, T}^{*}(t, x)=\mathrm{P}\left(t-X_{j: n}>x \mid T>t, X_{j: n}<t\right) \quad \text { for } j=1,2, \ldots, i .
$$

We now obtain the form of $P_{j, T}^{*}(t, x)$. Using the same steps as used to obtain the form of $P_{j, T}(t, x)$ earlier, we obtain, for $j \leq i-1$ and all $x, y \geq 0$,

$$
\begin{aligned}
\mathrm{P}\left(T>x, X_{j: n}<y\right) & =\sum_{k=i}^{n} \mathrm{P}\left(T=X_{k: n}, T>x, X_{j: n}<y\right) \\
& =\sum_{k=i}^{n} s_{k} \mathrm{P}\left(X_{k: n}>x, X_{j: n}<y\right) .
\end{aligned}
$$

Therefore, for all $x<t$ and $t>0$, we have

$$
\begin{aligned}
P_{j, T}^{*}(t, x) & =\frac{\mathrm{P}\left(t-X_{j: n}>x, T>t, X_{j: n}<t\right)}{\mathrm{P}\left(T>t, X_{j: n}<t\right)} \\
& =\sum_{k=i}^{n} p_{k}^{*}(t) \mathrm{P}\left(t-X_{j: n}>x \mid X_{k: n}>t, X_{j: n}<t\right),
\end{aligned}
$$

where

$$
p_{k}^{*}(t)=s_{k} \frac{\mathrm{P}\left(X_{k: n}>t, X_{j: n}<t\right)}{\mathrm{P}\left(T>t, X_{j: n}<t\right)}=\mathrm{P}\left(T=X_{k: n} \mid T>t, X_{j: n}<t\right) .
$$

Remark 3.1. As with $p_{k}(t)$ in Section 2 , we can easily show that

$$
p_{k}^{*}(t)=\frac{s_{k} W_{j, k}^{*}(t)}{\sum_{m=i}^{n} s_{m} W_{j, m}^{*}(t)},
$$

where $W_{j, m}^{*}(t)=\sum_{l=j}^{m-1}\left(\begin{array}{l}n \\ l\end{array}\right)(\phi(t))^{l}$ and $\phi(t)=F(t) / \bar{F}(t)$. Let $p^{*}(t)=\left(p_{1}^{*}(t), \ldots, p_{n}^{*}(t)\right)$. Using the form in (3.3), we can show, similar to Theorem 2.1 , that $\lim _{t \rightarrow 0} \boldsymbol{p}^{*}(t)=\boldsymbol{s}$ and $\lim _{t \rightarrow \infty} \boldsymbol{p}^{*}(t)=(0, \ldots, 0,1)$.

The following theorem can be established along the same lines as Theorem 2.2, and, hence, its proof is omitted for the sake of brevity.

Theorem 3.1. Let $\boldsymbol{p}^{*}(t)=\left(0,0, \ldots, 0, p_{i}^{*}(t), \ldots, p_{n}^{*}(t)\right)$, where $p_{k}^{*}(t)$ is as given in (3.3). Then

(a) $\boldsymbol{p}^{*}\left(t_{1}\right) \leq_{\text {st }} \boldsymbol{p}^{*}\left(t_{2}\right)$ for all $0 \leq t_{1} \leq t_{2}$;

(b) $p^{*}(t) \geq_{\text {st }} s$ for all $t \geq 0$.

In what follows, we present the main results of this section concerning some properties of the random variable

$$
\left(t-X_{j: n} \mid X_{k: n}>t, X_{j: n}<t\right)=\left(t-X_{j: n} \mid X_{j: n}<t<X_{k: n}\right) \quad \text { for } 1 \leq j<k \leq n .
$$

Let

$$
\gamma_{j, k, n}^{*}(t, x)=\mathrm{P}\left(t-X_{j: n}>x \mid X_{j: n}<t<X_{k: n}\right) .
$$


Then, by performing some algebra, it can be shown that

$$
\gamma_{j, k, n}^{*}(t, x)=\sum_{l=j}^{k-1} B_{j, l, n}^{*}(t, x) K_{l, j, k}^{* n}(t),
$$

where

$$
B_{j, l, n}^{*}(t, x)=\mathrm{P}\left(t-X_{j: n}>x \mid X_{l: n}<t<X_{l+1: n}\right)
$$

and

$$
\begin{aligned}
K_{l, j, k}^{* n}(t) & =\frac{\mathrm{P}\left(X_{l: n}<t<X_{l+1: n}\right)}{\mathrm{P}\left(X_{j: n}<t<X_{k: n}\right)} \\
& =\frac{\left(\begin{array}{c}
n \\
l
\end{array}\right)(F(t))^{l}(1-F(t))^{n-l}}{\sum_{m=j}^{k-1}\left(\begin{array}{l}
n \\
m
\end{array}\right)(F(t))^{m}(1-F(t))^{n-m}} \\
& =\frac{\left(\begin{array}{l}
n \\
l
\end{array}\right)(\phi(t))^{l}}{\sum_{m=j}^{k-1}\left(\begin{array}{l}
n \\
m
\end{array}\right)(\phi(t))^{m}}, \quad 1 \leq j \leq l<k \leq n .
\end{aligned}
$$

Remark 3.2. The quantity $B_{j, l, n}^{*}(t, x)$ can easily be shown to equal

$$
B_{j, l, n}^{*}(t, x)=\sum_{m=j}^{l}\left(\begin{array}{l}
l \\
m
\end{array}\right)\left(F_{t}(x)\right)^{m}\left(1-F_{t}(x)\right)^{l-m},
$$

where $F_{t}(x)=F(t-x) / F(t), 0<x<t$. Hence, from this equation we obtain the equality in distribution

$$
\left(t-X_{j: n} \mid X_{l: n}<t<X_{l+1: n}\right) \stackrel{\mathrm{D}}{=} X_{l-j+1: l}^{t},
$$

where $X_{l-j+1: l}^{t}$ denotes the $(l-j+1)$ th order statistic among $l$ i.i.d. random variables distributed as $(t-X \mid X<t)$ with distribution function $F_{t}(x)=F(t-x) / F(t)$.

It is well known (see Theorem 1.C.37 and Corollaries 1.C.38 and 1.C.39 of [23]) that

$$
\begin{gathered}
X_{j: m} \leq_{\operatorname{lr}} X_{i: n}, \quad j \leq i, m-j \geq n-i, \\
X_{k-1: m-1} \leq_{\operatorname{lr}} X_{k: m}, \quad k=2,3, \ldots, m, \\
X_{k: m-1} \geq_{\operatorname{lr}} X_{k: m}, \quad k=1,2, \ldots, m-1 .
\end{gathered}
$$

Hence, we have

$$
X_{l-j+1: l}^{t} \leq \operatorname{lr} X_{l+1-j+1: l+1}^{t}, \quad X_{l-j+1: l}^{t} \leq_{\operatorname{lr}} X_{l-j+1: l-1}^{t}=X_{l-1-(j-1)+1: l-1}^{t} .
$$

This, in turn, implies that

$$
\begin{aligned}
& \left(t-X_{j: n} \mid X_{l: n}<t<X_{l+1: n}\right) \leq \operatorname{lr}\left(t-X_{j: n} \mid X_{l+1: n}<t<X_{l+2: n}\right), \\
& \left(t-X_{j: n} \mid X_{l: n}<t<X_{l+1: n}\right) \leq \operatorname{lr}\left(t-X_{j: n} \mid X_{m: n}<t<X_{m+1: n}\right), \quad l \leq m, \\
& \left(t-X_{j: n} \mid X_{l: n}<t<X_{l+1: n}\right) \leq_{\operatorname{lr}}\left(t-X_{j-1: n} \mid X_{l-1: n}<t<X_{l: n}\right) .
\end{aligned}
$$

Remark 3.3. Asadi [1] showed that

$$
\mathrm{P}\left(t-X_{j: l}>x \mid X_{l: l}<t\right)=\sum_{m=j}^{l}\left(\begin{array}{c}
l \\
m
\end{array}\right)\left(F_{t}(x)\right)^{m}\left(1-F_{t}(x)\right)^{l-m} .
$$


Hence, from (3.4) we obtain

$$
\left(t-X_{j: n} \mid X_{l: n}<t<X_{l+1: n}\right) \stackrel{\mathrm{D}}{=} X_{l-j+1: l}^{t} \stackrel{\mathrm{D}}{=}\left(t-X_{j: l} \mid X_{l: l}<t\right),
$$

and so

$$
\begin{aligned}
& \left(t-X_{j: l} \mid X_{l: l}<t\right) \leq \operatorname{lr}\left(t-X_{j: l+1} \mid X_{l+1: l+1}<t\right), \\
& \left(t-X_{j: l} \mid X_{l: l}<t\right) \leq \operatorname{lr}\left(t-X_{j: m} \mid X_{m: m}<t\right), \quad l \leq m .
\end{aligned}
$$

We now present a theorem that is useful for our subsequent derivations. The proof of the theorem is similar to that of Theorem 2.4 and is therefore omitted for the sake of brevity.

Theorem 3.2. If $k \leq m$ then $\left(t-X_{j: n} \mid X_{j: n}<t<X_{k: n}\right) \leq \operatorname{lr}\left(t-X_{j: n} \mid X_{j: n}<t<X_{m: n}\right)$.

Theorem 3.3. Let $\boldsymbol{p}_{1}^{*}(t)$ and $\boldsymbol{p}_{2}^{*}(t)$ for a fixed $t>0$ be the vectors of coefficients in representation (3.2), of two coherent systems of order $n$, both based on components with i.i.d. lifetimes having a common continuous distribution function $F$. Let $T_{1}$ and $T_{2}$ denote the respective lifetimes of the two systems. We then have the following stochastic inequalities:

(a) if $\boldsymbol{p}_{1}^{*}(t) \leq_{\mathrm{st}} \boldsymbol{p}_{2}^{*}(t)$ then $\left(t-X_{j: n} \mid X_{j: n}<t<T_{1}\right) \leq_{\mathrm{st}}\left(t-X_{j: n} \mid X_{j: n}<t<T_{2}\right)$;

(b) if $\boldsymbol{p}_{1}^{*}(t) \leq_{\mathrm{rh}} \boldsymbol{p}_{2}^{*}(t)$ then $\left(t-X_{j: n} \mid X_{j: n}<t<T_{1}\right) \leq_{\mathrm{rh}}\left(t-X_{j: n} \mid X_{j: n}<t<T_{2}\right)$;

(c) if $\boldsymbol{p}_{1}^{*}(t) \leq \operatorname{lr} \boldsymbol{p}_{2}^{*}(t)$ then $\left(t-X_{j: n} \mid X_{j: n}<t<T_{1}\right) \leq_{\operatorname{lr}}\left(t-X_{j: n} \mid X_{j: n}<t<T_{2}\right)$.

Proof. The theorem can be proved by using Theorem 1.B.12 of [23], and proceeding along the same lines as those used to establish Theorem 2.3.

\subsection{Ageing properties of the failed components}

In this subsection we study some ageing properties of $P_{j, T}^{*}(t)$. First, we note that $B_{j, l, n}^{*}(t, x)$ can be rewritten as

$$
\begin{aligned}
H_{l}^{* j}(t, x) & =\sum_{m=j}^{l}\left(\begin{array}{c}
l \\
m
\end{array}\right)\left(F_{t}(x)\right)^{m}\left(1-F_{t}(x)\right)^{l-m} \\
& =\int_{0}^{F(t-x) / F(t)} j\left(\begin{array}{l}
l \\
j
\end{array}\right) u^{j-1}(1-u)^{l-j} \mathrm{~d} u .
\end{aligned}
$$

Let $r(t)=f(t) / F(t)$ be the reversed hazard rate of the components of the system. Then it is easy to see that $r(t)$ is decreasing if and only if $F(t-x) / F(t)$ is an increasing function of $t, t>0$. Hence, from (3.5), $r(t)$ is decreasing if and only if $B_{j, l, n}^{*}(t, x)$ is an increasing function of $t$ for all $x \geq 0$. The above discussion leads to the following lemma.

Lemma 3.1. Let $r(t)$, the reversed hazard rate of the components of the system, be decreasing in $t$ for $t>0$. Then, $\gamma_{j, k, n}^{*}(t, x)$ is an increasing function of $t$.

Proof. Note that

$$
\frac{\mathrm{d}}{\mathrm{d} t} \gamma_{j, k, n}^{*}(t, x)=\sum_{l=j}^{k-1}\left(\frac{\mathrm{d}}{\mathrm{d} t} H_{l}^{* j}(t, x)\right) K_{l, j, k}^{n}(t)+\sum_{l=j}^{k-1} H_{l}^{* j}(t, x)\left(\frac{\mathrm{d}}{\mathrm{d} t} K_{l, j, k}^{n}(t)\right) .
$$

The assumption that $r(t)$ is decreasing implies, from the discussion above, that the first term on the right-hand side of the above equation is nonnegative. The second term can also be shown to be nonnegative if we follow the same arguments as used to show that the second term on the right-hand side of (2.8) (in Lemma 2.6) is nonnegative. This completes the proof. 
The following theorem extends the result of Lemma 3.1 to coherent systems. First, since

$$
\left(t-X_{j: n} \mid X_{j: n}<t<X_{k: n}\right) \leq_{\mathrm{st}}\left(t-X_{j: n} \mid X_{j: n}<t<X_{k+1: n}\right)
$$

we have

$$
\gamma_{j, k, n}^{*}(t, x) \leq \gamma_{j, k+1, n}^{*}(t, x)
$$

Theorem 3.4. Let $r(t)$, the reversed hazard rate of the components of the system, be decreasing in $t$ for $t>0$. Then, $P_{j, T}^{*}(t)$ is an increasing function of $t$.

Proof. Note that

$$
\frac{\mathrm{d}}{\mathrm{d} t} P_{j, T}^{*}(t, x)=\sum_{k=i}^{n}\left(\frac{\mathrm{d}}{\mathrm{d} t} \gamma_{j, k, n}^{*}(t, x)\right) p_{k}^{*}(t)+\sum_{k=i}^{n} \gamma_{j, k, n}^{*}(t, x)\left(\frac{\mathrm{d}}{\mathrm{d} t} p_{k}^{*}(t)\right) .
$$

The first term on the right-hand side is nonnegative due to Lemma 3.1. The second term can also be proved to be nonnegative by following similar lines to those used in the proof of Theorem 2.4 and by using inequality (3.6).

\subsection{Stochastic comparison between the failed components}

Let $X$ and $Y$ denote two continuous random variables with distribution functions $F$ and $G$, density functions $f$ and $g$, and reversed hazard rates $r_{F}$ and $r_{G}$, respectively. Consider two coherent systems with the same signature as in (3.1), and assume that $T_{1}$ and $T_{2}$ denote the lifetimes of the systems whose components are distributed as $F$ and $G$, respectively. In this subsection we prove that when the components of the system are ordered in terms of reversed hazard rates, then the inactivity times of the failed components of the systems are stochastically ordered. First, let $X_{i: n}$ and $Y_{i: n}, i=1,2, \ldots, n$, denote the ordered lifetimes of the two systems, respectively. From (3.5), it can be easily shown that $X \leq_{\text {rh }} Y$ if and only if

$$
\left(t-X_{j: n} \mid X_{l: n}<t<X_{l+1: n}\right) \geq_{\mathrm{st}}\left(t-Y_{j: n} \mid Y_{l: n}<t<Y_{l+1: n}\right) .
$$

If we follow the same arguments as those used to prove Lemma 2.8, we can show that if $X \leq_{\mathrm{rh}} Y$ then

$$
\left(t-X_{j: n} \mid X_{j: n}<t<X_{k: n}\right) \geq_{\mathrm{st}}\left(t-Y_{j: n} \mid Y_{j: n}<t<Y_{k: n}\right) .
$$

Finally, the steps that were used to prove Theorem 2.5 lead to the following theorem, which we therefore present here without a proof for the sake of brevity.

Theorem 3.5. Let $X \leq_{\mathrm{rh}} Y$. Then $\left(t-X_{j: n}>x \mid X_{j: n}<t<T_{1}\right) \geq_{\mathrm{st}}\left(t-Y_{j: n}>x \mid Y_{j: n}<\right.$ $\left.t<T_{2}\right)$.

\section{Acknowledgements}

The authors wish to thank an anonymous referee for some useful comments and suggestions on an earlier version of this manuscript, which resulted in this improved version. M. Asadi's work was supported in part by the Ordered and Spatial Data Center of Excellence of Ferdowsi University of Mashhad. 


\section{References}

[1] Asadi, M. (2006). On the mean past lifetime of components of a parallel system. J. Statist. Planning Infer. 136, 1197-1206.

[2] Asadi, M. and Bayramoglu, I. (2005). A note on the mean residual life function of a parallel system. Commun. Statist. Theory Meth. 34, 475-484.

[3] Asadi, M. and Bayramoglu, I. (2006). The mean residual life function of a $k$-out-of- $n$ structure at the system level. IEEE Trans. Reliab. 55, 314-318.

[4] Asadi, M. and Berred, A. (2011). Properties and estimation of mean past lifetime. Statistics (electronic).

[5] Asadi, M. And Goliforushani, S. (2008). On the mean residual life function of coherent systems. IEEE Trans. Reliab. 57, 574-580.

[6] Bairamov, I., Ahsanullah, M. and Akhundov, I. (2002). A residual life function of a system having parallel or series structures. J. Statist. Theory Appl. 1, 119-131.

[7] Gertsbakh, I., Shpungin, Y. and Spizzichino, F. (2011). Signatures of coherent systems built with separate modules. J. Appl. Prob. 48, 843-855.

[8] Khaledi, B. E. ANd Shaked, M. (2007). Ordering conditional lifetimes of coherent systems. J. Statist. Planning Infer. 137, 1173-1184.

[9] Kochar, S., Mukerjee, H. and Samaniego, F. J. (1999). The 'signature' of a coherent system and its application to comparisons among systems. Naval Res. Logistics 46, 507-523.

[10] LI, S. AND LYNCH, J. (2010). Some elementary ideas concerning the complexity of system structure. Naval Res. Logistics 57, 626-633.

[11] Li, X. And Zhang, Z. (2008). Some stochastic comparisons of conditional coherent systems. Appl. Stoch. Models Business Industry 24, 541-549.

[12] LI, X. AND Zhang, Z. (2008). Stochastic comparisons on general inactivity time and general residual life of $k$-out-of-n systems. Commun. Statist. Simul. Comput. 37, 1005-1019.

[13] Li, X. And ZhaO, P. (2006). Some ageing properties of the residual life of $k$-out-of- $n$ systems. IEEE Trans. Reliab. 55, 535-541.

[14] Mahmoudi, M. AND Asadi, M. (2011). On the conditional signature of coherent systems. IEEE Trans. Reliab. 60, $817-822$.

[15] Navarro, J. and Hernandez, P. J. (2008). Mean residual life functions of finite mixtures, order statistics and coherent systems. Metrika 67, 277-298.

[16] Navarro, J., Balakrishnan, N. And Samaniego, F. J. (2008). Mixture representations of residual lifetimes of used systems. J. Appl. Prob. 45, 1097-1112.

[17] Navarro, J., Ruiz, J. M. and Sandoval, C. J. (2005). A note on comparisons among coherent systems with dependent components using signatures. Statist. Prob. Lett. 72, 179-185.

[18] Navarro, J., Ruiz, J. M. and Sandoval, C. J. (2007). Properties of coherent systems with dependent components. Commun. Statist. Theory Meth. 36, 175-191.

[19] Navarro, J., Samaniego, F. J. and Balakrishnan, N. (2011). The joint signature of coherent systems with shared components. J. Appl. Prob. 47, 235-253.

[20] Navarro, J., Samaniego, F. J. And Balakrishnan, N. (2011). Signature-based representations for the reliability of systems with heterogeneous components. J. Appl. Prob. 48, 856-867.

[21] Samaniego, F. J. (1985). On closure of the IFR class under formation of coherent systems. IEEE Trans. Reliab. 34, 69-72.

[22] Samaniego, F. J., Balakrishnan, N. and Navarro, J. (2009). Dynamic signatures and their use in comparing the reliability of new and used systems. Naval Res. Logistics 56, 577-591.

[23] Shaked, M. and Shanthikumar, J. G. (2007). Stochastic Orders. Springer, New York.

[24] Tavangar, M. And Asadi, M. (2010). A study on the mean past lifetime of the components of $(n-k+1)$-out-of- $n$ system at the system level. Metrika 72, 59-73.

[25] Zhang, Z. (2010). Mixture representations of inactivity times of conditional coherent systems and their applications. J. Appl. Prob. 47, 876-885.

[26] Zhang, Z. (2010). Ordering conditional general coherent systems with exchangeable components. J. Statist. Planning Infer. 140, 454-460. 